\title{
Observing Quantum Vacuum Lensing in a Neutron Star Binary System
}

\author{
Arnaud Dupays, Cécile Robilliard, and Carlo Rizzo \\ Laboratoire Collisions, Agrégats, Réactivité, IRSAMC, CNRS/UPS, 31062 Toulouse, France \\ Giovanni F. Bignami \\ Centre d'Etude Spatiale des Rayonnements, CNRS/UPS, 31401 Toulouse, France \\ Dipartimento di Fisica Nucleare e Teorica, Universita di Pavia, Italy
}

(Dated: October 28, 2018)

\begin{abstract}
In this letter we study the propagation of light in the neighbourhood of magnetised neutron stars. Thanks to the optical properties of quantum vacuum in the presence of a magnetic field, light emitted by background astronomical objects is deviated, giving rise to a phenomenon of the same kind as the gravitational one. We give a quantitative estimation of this effect and we discuss the possibility of its observation. We show that this effect could be detected by monitoring the evolution of the recently discovered double neutron star system J0737-3039.
\end{abstract}

PACS numbers:

Keywords:

Neutron stars have been intensively studied since their discovery as pulsars, i.e. stars with a magnetic dipole field tilted with respect to their rotation axis, emitting radio waves along their dipole axis as the star spins [1]. Typically their mass is on the order of that of the Sun, and their radius of the order of $10 \mathrm{~km}$. Their spinning period ranges from tens of milliseconds for young stars to seconds, decreasing with star age. The magnetic field of neutron stars can be as high as $10^{11} \mathrm{~T}$ as in the case of magnetars 2] while magnetic fields of the order of $10^{8}-10^{9} \mathrm{~T}$ are more common. Most of the known pulsars are isolated stars, less than 100 are known to be in binary systems with non-degenerate stars, few $(\sim 5)$ are in neutron star-neutron star binary systems. Very recently, a specially interesting NS-NS system (J0737-3039) has been discovered [3].

On the other hand, it is known that quantum vacuum is a non linear optical medium [4]. In the seventies it was clearly pointed out [5] that the velocity of light propagating in a quantum vacuum in the presence of a magnetic field changes of a quantity which depends on the polarization of light with respect to the magnetic field direction. Those calculations were prompted by early speculations that neutron stars could be a source of very intense magnetic fields. Since then, most authors have been interested in how the magnetic field can affect the propagation of radiation emitted by the neutron star itself [7].

In this letter we investigate the case of radiation emitted by another astronomical object passing close enough to the NS to be significantly affected by its magnetic field. The case in point will be seen to be that of J0737, especially in the context of its orbital evolution.

In the vicinity of a NS, owing to the optical properties of quantum vacuum in the presence of a magnetic field, light emitted by astronomical sources is deviated, giving rise to a phenomenon of the same kind as the gravita- tional one predicted by general relativity. We first prove that fields of the order of $10^{9} \mathrm{~T}$ are not sufficient to give an effect big enough to be detected in the case of isolated NS or in the case of NS-non-degenerate star systems. On the other hand we show that quantum vacuum effect could be observed by monitoring the evolution of the recently discovered NS-NS system J0737-3039.

It is known that light generally bends towards regions with a higher index of refraction [6]. Because of refraction index variation in magnetized quantum vacuum, light passing near a magnetar behaves as if it were attracted by the magnetar and it should bend considerably, giving rise to a lensing effect. Actually, since the velocity of light depends on its polarization, the cosmos around a magnetar acts as a birefringent medium and different polarizations should bend differently.

In the presence of a static magnetic field $\mathbf{B}$ the index of refraction for light polarized parallel (resp. perpendicular) to the magnetic field $n_{\|(\perp)}$ can be written as $\underline{5}$ ]

$$
n_{\|(\perp)}=1+a_{\|(\perp)} B^{2}
$$

where $a_{\|}$is about $9 \times 10^{-24} \mathrm{~T}^{-2}$ and $a_{\perp}$ is about $5 \times$ $10^{-24} \mathrm{~T}^{-2}$. Deviations from these formulae appear for magnetic field values bigger than the critical field $4.410^{9}$ $\mathrm{T}$ [10] when a slower dependence on $B$ sets in. However, in our case, since we are interested in subcritical fields, such deviation is negligible. This variation of the index of refraction of light in vacuum is very small when the magnetic field applied is the order of the fields that can be produced in a terrestrial laboratory (about $10 \mathrm{~T}$ ), so that this effect has not yet been detected [9]. For our purpose, this effect can be considered achromatic because this formula applies up to photon energies much larger than optical energies [1].

For sake of simplicity, in what follows we will neglect intrinsic polarization of light, considering that astronom- 
ical sources are weakly polarized.

Propagation of light can be studied using the differential equation of light rays $[$. The specific case of light propagating in a plane perpendicular to the NS magnetic dipole moment has been treated in ref. [8]. In ref. [8] it was concluded that a neutron star with a magnetic field of around $10^{9} \mathrm{~T}$ could give a detectable effect.

In Fig. 1] we present the results of our calculations of the deviation angle of light ray for different orientations of the magnetic dipole moment, in the case of a magnetic field of $10^{9} \mathrm{~T}$. This shows that the deviation angle goes as $1 / \rho^{6}$, where $\rho$ is the minimal distance between the light ray and the neutron star.

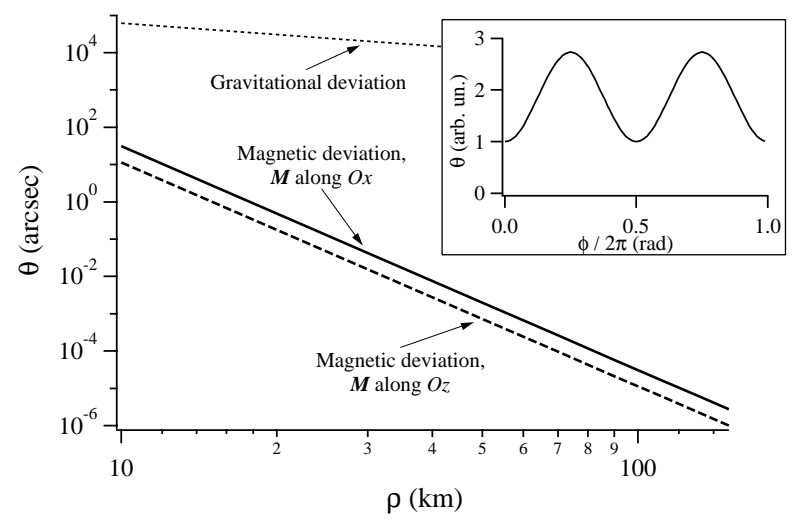

FIG. 1: Deviation angle of a light ray propagating parallel to the $\mathrm{Oz}$ axis with a minimal distance $\rho$ to the center of the NS, for two different orientations of the magnetic dipole moment $M$. The value of the magnetic field at the surface of the star is $B_{0}=10^{9} \mathrm{~T}$. Inset shows deviation modulation with NS rotational phase.

This figure also shows that the deviation angle depends on the orientation of the magnetic moment. Since the neutron star spins, the light deviation varies in time at twice the frequency of the spinning pulsar, owing to the spatial symmetry of the dipole field. Depending on orientation, the modulation of the effect can be as high as $40 \%$. Magnetic deviations at very short distances from the neutron star become of the same order as the gravitational one only in the case of magnetar $\left(B_{0}=10^{11} \mathrm{~T}\right)$, i.e $\theta \sim 10^{5}$ arcsec. In the more common cases of a field $B_{0}=10^{8}-10^{9} \mathrm{~T}$, the magnetic deviation is always very small compared to the gravitational one (see Fig. 1). On the other hand, for astronomical distances, even very small deflection can completely change observations. It is also important to note that magnetic birefringence exists in any medium (Cotton-Mouton effect), and in particular in gases. The residual gas that fills the space around neutron stars is also magnetically birefringent. The density of this gas is between 1 and $10^{4}$ particles per $\mathrm{cm}^{3}$. Thanks to the value of the Cotton-Mouton constant for simple gases like hydrogen [12, the effect induced by this quantity of gas will be at least 6 orders of magnitude smaller that the one induced by the quantum vacuum. This effect will thus be neglected here.

To study the feasibility of the observation of quantum vacuum mirage, we started with the case of an isolated neutron star with a distant background source (see Fig. 2). To estimate the quantum vacuum lensing, we have performed numerical calculations. Following the method proposed by Paczyński [13] for gravitational lensing, we have included the quantum vacuum effect in the total angular deviation of light rays. The problem consists of calculating the imaging of a distant source to detect modifications to the magnification due to gravitational lensing. For sake of simplicity we have considered the case of a magnetic dipole moment parallel to the light propagation direction. The deflection is then given by :

$$
\theta=\frac{4 G M}{\rho c^{2}}+\frac{5 \pi a B_{0}^{2} \rho_{0}^{6}}{\rho^{6}}
$$

where $G$ is the gravitational constant, $M$ and $\rho_{0}$ the mass and the radius of the pulsar respectively, and $B_{0}$ the value of the magnetic field at the surface of the star. In our simulations $a$ has been taken equal to $a_{\perp}$. Finally, $\rho$ is the distance from the deflected light ray to the center of the pulsar. Fig. 2 presents the geometry of the system, projected on the trajectory plane of the light ray. The distances from the observer to the lens (pulsar) and to the source are indicated as $D_{L}$ and $D_{S}$, respectively. $\rho_{S}$ is the distance from the pulsar to the line of sight.

Defining $D=\left(D_{L}-D_{S}\right) D_{L} / D_{S}$, the linear Einstein ring radius is then given by $R_{E}=\sqrt{4 G M D / c^{2}}$. For a distance from the pulsar to the line of sight $\rho_{S}$ larger than $R_{E}$, the gravitational lensing is very small 13. With $M \sim M_{\odot}, \rho_{0} \sim 10 \mathrm{~km}$ and $B_{0}=10^{8}-10^{9} \mathrm{~T}$ (which corresponds to a reasonable value of the magnetic field at the surface of a neutron star), the calculations show that $\rho_{S} \gg R_{E}$ : the magnification is then negligible for both gravitational and total lensing. The next case to be studied was a binary system consisting of a neutron star with a normal star companion. With respect to Fig. 2] $D$ changes with the orbital trajectory, but the result is similar to the previous case. To be able to observe any modification of the gravitational lensing due to magnetic effects of the order of few percent, the latter have to occur at distances of the order of $R_{E}$. These conditions begin to be realized for a totally non realistic magnetic field $B_{0} \sim 10^{16} \mathrm{~T}$. The effect of $10^{8} \mathrm{~T}$ corresponds to negligible contributions. Our results are here in disagreement with the conclusions of authors of ref. [8]. In any case, in a realistic astronomical case, the effects of accretion onto the NS of mass transferred from the companion will completely obliterate subtle effects of the type we are interested in.

Much more interesing appears to be the case of the NS-NS binary J0737. No accretion perturbs this system which, furthermore, presents a very favourable orbital inclination (currently, $i \sim 87^{\circ}$ ) 15. In fact, orbital pre- 


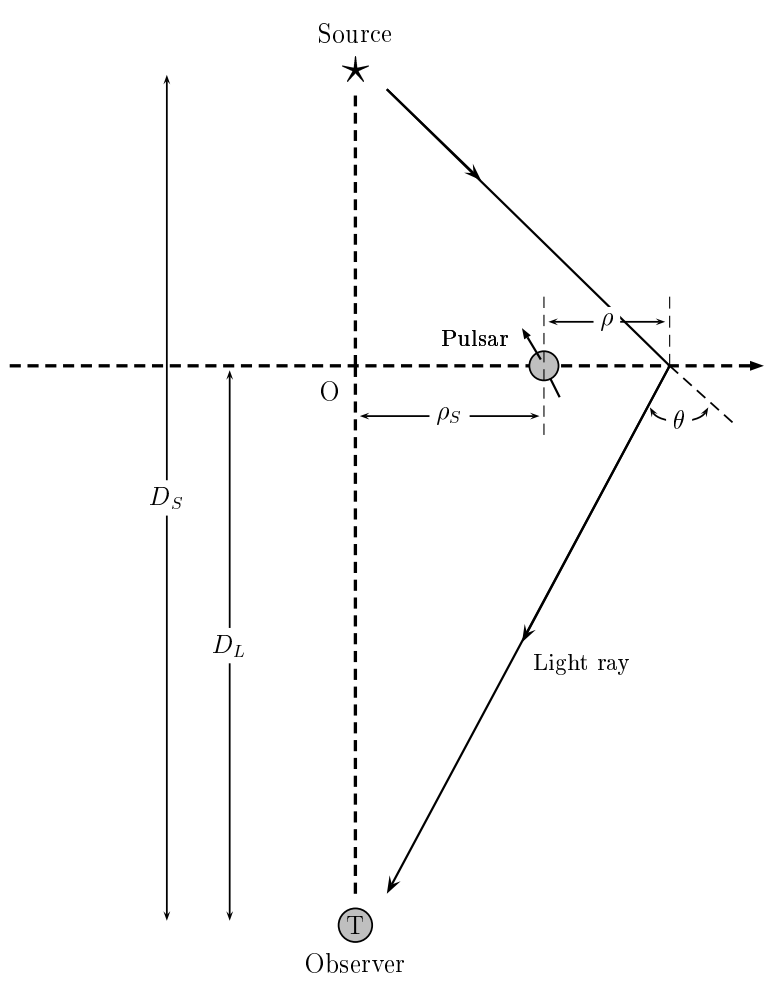

FIG. 2: Geometry of the deflection effect. The light rays are deflected near the pulsar by the angle $\theta$.

cession of the system allows one to predict that, before $2020, i$ will be extremely close to $90^{\circ}$. The two NS will actually geometrically "eclipse" each other, as seen by the earth, for a short time. In any case they will remain practically aligned for several revolutions of their 2 hours and 45 minutes orbit during a time long enough to allow for meaningful observations.

Nowadays the radio beam, emitted by neutron star A, passes at around $20000 \mathrm{~km}$ from the surface of neutron star B. The beam is attenuated or deviated in such a way that eclipses are visible from the earth. Recently, a modulation of the beam at twice the spinning frequency of the neutron star B has been observed 16]. The explanation of this effect is the interaction between light and the magnetosphere of star B. We also know that this absorption should disappear for photons above about 7.5 $\mathrm{GHz}$ 17]. The main difference between this case and the previous two is that the radiation emitted by neutron star A can be treated as a single directional light ray. We can thus directly observe the lensing effect due to gravitational and magnetic deflections of light by pulsar B.

In Fig. [3and Fig. 4 we present the results of our calculations. The relative flux density of pulsar A arriving on Earth for different values of $i$ versus time is shown Fig. 3] The calculations assume photons to be energetic enough not to interact with the magnetosphere of pulsar B. The

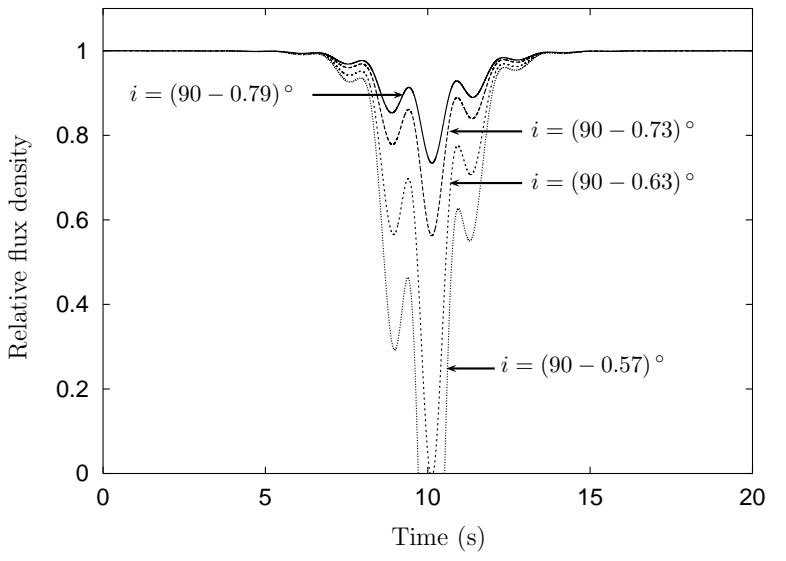

FIG. 3: Relative flux density of pulsar A versus time for different values of the orbital inclination $i$. The value of the magnetic field at the surface of pulsar $\mathrm{B}$ is $B_{0}=10^{8} \mathrm{~T}$. Flux densities have been normalized to unity when the magnetic effect is negligible. Gravitational lensing is taken into account.

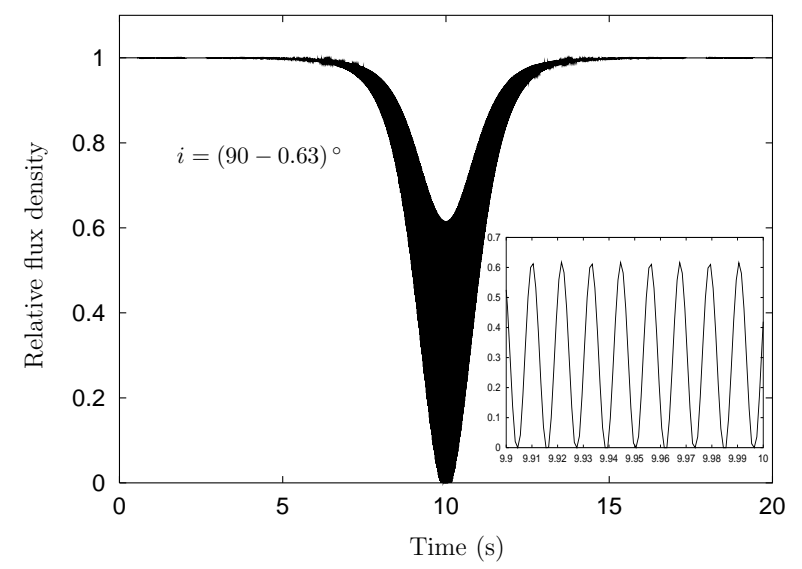

FIG. 4: Relative flux density of pulsar B versus time for an orbital inclination $i=(90-0.63)^{\circ}$. The value of the magnetic field at the surface of pulsar $\mathrm{A}$ is $B_{0}=10^{8} \mathrm{~T}$. Flux densities have been normalized to unity when the magnetic effect is negligible. Gravitational lensing is taken into account. Inset shows the small modulation period of the signal $(\sim 11.5 \mathrm{~ms})$. This modulation is so rapid that it appears as a continuum in the main figure.

results have been obtained using the geometry data published in [15. The modulation shown by the signal is due to the spin period of $2.77 \mathrm{~s}$ of pulsar B. Since the spin period of pulsar A is smaller than the one of pulsar B (23 ms), pulsar A will produce a similar effect on the flux density of pulsar B, but with the smaller modulation period (see Fig. 4). Total eclipse occurs when the bending is so large that the beam no longer intersect the line of sight. Thus, quantum vacuum lensing will give an effect similar to the radio eclipses observed by [16], of course on photons which should not be eclipsed. Thanks 
to the fact that quantum vacuum lensing is achromatic, this also applies to photons of higher energy, such as Xrays, also observed from this system 18. Any observation, once $B_{0}$ is known, will provide the value of $a$, which has never been measured. A measure of $a$ could confirm the classical QED predictions given in [4]. However, we note that magnetic birefringence could also be induced by physics beyond the standard model, as in the case of the existence of axions, as shown in [19].

In conclusion, we have shown that the magneto-optical properties of the quantum vacuum induced by the very high magnetic field in the vicinity of a neutron star can affect the propagation of light passing near such a pulsar. It will give rise to a lensing effect that could be detected on earth by monitoring the double neutron star system J0737-3039, recently discovered. Its observation would consitute the first experimental confirmation of quantum vacuum optical properties. Thus, a continuous monitoring of this very peculiar system will be valuable to test quantum electrodynamic theory, and beyond.

[1] A. Lyne, F. Graham-Smith, 1989, Pulsar Astronomy, Cambridge U.P

[2] A.I. Ibrahim, J.H. Swank and W. Parke, ApJL, 584, L17 (2002); G. F. Bignami, P. A. Caraveo, A. De Luca and S. Mereghetti, Nature 423, 725 (2003).
[3] M. Burgay, N. D'Amico, Possenti et al., Nature 426, 531 (2003).

[4] H. Euler and B. Kockel, Naturwiss. 23, 246 (1935); W. Heisenberg and H. Euler, Z. Phys. 98, 714 (1936).

[5] Z. Bialynicka-Birula and I. Bialynicka-Birula, Phys. Rev. D 2, 2341 (1970).

[6] M. Born and E. Wolf, Principles of Optics, Pergamon Press, New York (1983).

[7] see e.g. J. S. Heyl and N. J. Shaviv, Phys. Rev. D, 66, 023002 (2002); D. Lai and W.C.G. Ho, Phys. Rev. Lett. 91, 071101 (2003); H.J. Mosquera Cuesta, J.A. de Freitas Pacheco and J.M. Salim, arXiv:astro-ph/0408152 1 9 Aug 2004.

[8] V.I. Denisov, I.P. Denisova, and S.I. Svertilov, Doklady Physics 46, 754 (2001).

[9] E. Zavattini, Comment. At. Mol. Phys., 3383 (1996).

[10] J.S. Heyl and L. Hernquist, J. Phys. A: Math. Gen. 30, 6485 (1997).

[11] W. Tsai and T. Erber, Phys. Rev. D, 12, 1132 (1975).

[12] C. Rizzo, A. Rizzo and D.M. Bishop, Int. Rev. in Phys. Chem. 16, 81 (1997).

[13] B. Paczyński, Ann. Rev. Astron. \& Ap., 34, 419 (1996).

[14] C. Alcock et al. Nature 365621 (1993); E.Aubourg et al. ibidem 623 .

[15] F.A. Jenet and S.M. Ransom, Nature 428919 (2004).

[16] M.A. McLaughlin et al., ApJ letters, in press (2004).

[17] Kaspi et al., ApJL, 613 L137 (2004).

[18] A. Pellizzoni et al., ApJ, 612 L49 (2004).

[19] L. Maiani, R. Petronzio, and E. Zavattini, Phys. Lett. B, 175, 359 (1986). 